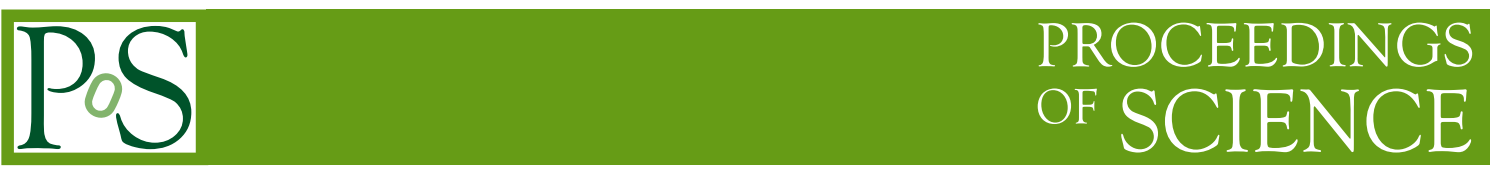

\title{
Aspects of heavy flavor jet physics in heavy ion collisions
}

\author{
Ivan Vitev* \\ Los Alamos National Laboratory, Theoretical Division, Mail Stop B283, Los Alamos, NM 87544 \\ E-mail: ivitev@lanl.gov
}

\begin{abstract}
In these proceedings I discuss several recent developments in the physics of heavy flavor jets in heavy ion collisions. i) The dijet mass modification in nucleus-nucleus reactions has been proposed as a new observable with enhanced sensitivity to parton energy loss in nuclear matter. It also enables more precise studies of heavy quark mass effects on parton shower formation. ii) Computational techniques from soft-collinear effective theory have allowed us to bridge the gap between high energy and heavy ion QCD phenomenology. I show the first application of the semiinclusive jet function formalism to heavy flavor jet production in proton-nucleus and nucleusnucleus collisions at the LHC. iii) Last but not least, central to the theoretical calculations of heavy flavor jets is the accurate theoretical description of in-medium parton showers. A formalism to compute branching processes in nuclear matter to any desired order in opacity has been developed and illustrative numerical results are presented.
\end{abstract}

13th International Workshop in High pT Physics in the RHIC and LHC Era (High-pT2019)

19-22 March 2019

Knoxville, Tennessee, USA

${ }^{*}$ Speaker. 


\section{Introduction}

Theoretical and experimental studies of heavy flavor are central to high-energy nuclear physics. They provide new avenues to explore Quantum Chromodynamics and new diagnostics of the transport properties of nuclear matter matter [1]. In these proceedings I will focus on open heavy flavor and jets that contain charm $(c)$ and beauty $(b)$ quarks in particular. Theoretical investigation of heavy-flavor tagged jet production in heavy ion collisions has been somewhat limited [2, 3, 4, 5], even though experimental effort in this direction is ramping up [6, 7, 8, 9]. At transverse momenta $p_{T} \geq 100 \mathrm{GeV}$ the suppression of $b$-jets in nucleus-nucleus $(\mathrm{A}+\mathrm{A})$ reactions relative to the protonproton $(p+p)$ baseline was found to be similar to the one for inclusive jets. In the quest to identify observables that are sensitive to the heavy heavy quark mass, groomed soft-dropped momentum sharing distributions in heavy ion collisions show promise at transverse momenta $\leq 50 \mathrm{GeV}$ [4]. An alternative strategy is to exploit the fact that multi-jet events are abundant in high energy hadronic collisions and devise observables, such as dijet mass, that amplify the effects of quenching [10].

Until recently, calculations of heavy flavor jet observables were limited to the traditional charm and beauty quark energy loss approach, see for example [11]. It is now possible to evaluate $b$-jet and $c$-jet cross sections using the technique of semi-inclusive jet functions [12]. This theoretical development was enabled by the recent derivation of the semi-inclusive jet functions for heavy flavor jets in the vacuum [13], and the in-medium splitting kernels for heavy flavor to first order in opacity [14]. Phenomenological results give a good description of the LHC experimental measurements with improved control over theoretical uncertainties. Effective field theories for heavy jet substructure are also being developed, first in $p+p$ collisions [15]. This serves as a motivation to revisit and further improve the Altarelli-Parisi splitting functions in nuclear matter that are the key ingredients in high-precision calculations in the theory of strong interactions and in Monte-Carlo event generators for heavy ion physics [16]. The evaluation of correlation effects due to coherent multiple scattering on parton branching processes is discussed.

These developments are outlined below in more detail: the dijet mass calculations are shown in Section 2. Section 3 summarizes the semi-inclusive jet function approach to $b$ - and $c$-jets. Parton branching calculations beyond the soft gluon emission limit to higher order in opacity follow in Section 4. Conclusions are given in Section 5.

\section{Back-to-back dijets and dijet mass modification}

Back-to-back light and heavy flavor dijet measurements are important for the accurate study of jet production and propagation in a dense QCD medium. They can test the path length, color charge, and mass dependence of quark and gluon energy loss in the quark-gluon plasma (QGP) produced in ultra-relativistic nucleus collisions. Traditional studies of dijets in heavy ion physics have focused on observables such as the dijet momentum imbalance shift - the normalized distribution of the imbalance variable $z_{J}=p_{2 T} / p_{1 T}$. In the absence of a QGP one expects that the transverse momenta of the two jets are approximately balanced. On the other hand, in heavy ion collisions one of the jets may lose more energy than the other, resulting in a downshift of the peak in $z_{J}$ distribution because of strong in-medium interactions. This is shown in the left panel of Figure 1, 

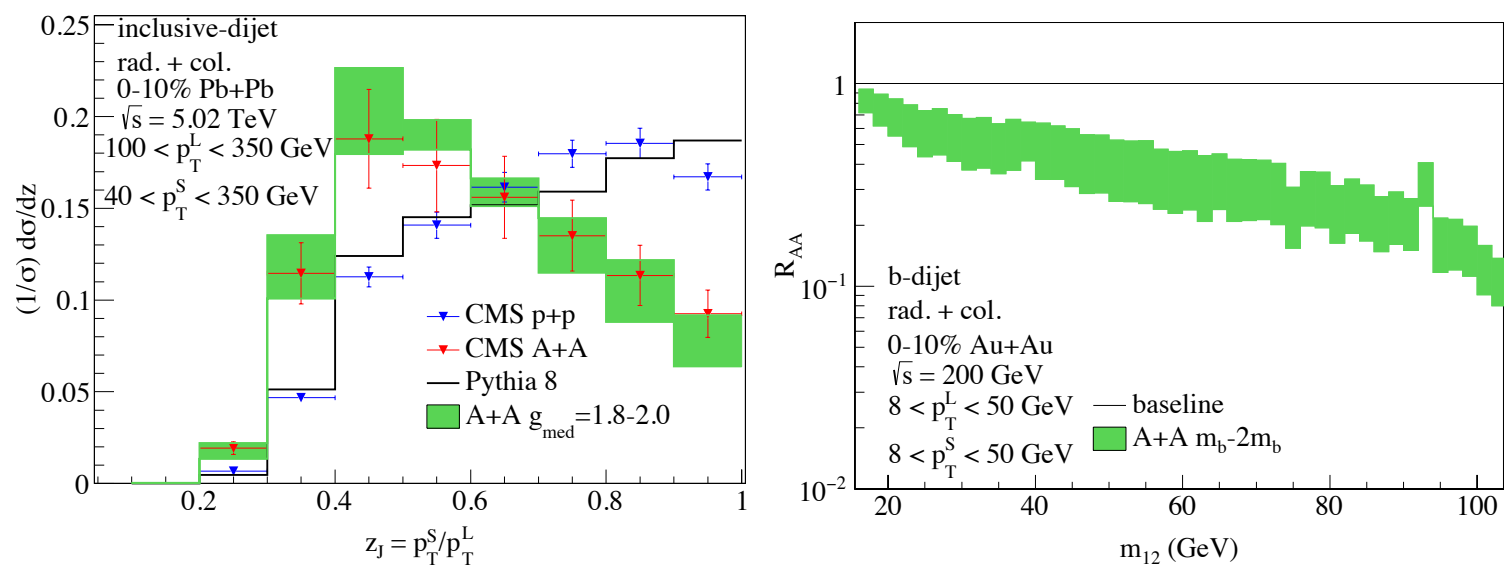

Figure 1: Left: the dijet imbalance $z_{J}$ distributions for inclusive jets at $\sqrt{s_{N N}}=5.02 \mathrm{TeV}$ are compared to CMS collaboration data [7]. The band corresponds to a range of coupling strengths between the jet and the medium: $g_{\text {med }}=1.8-2.0$. Right: nuclear modification factor plotted as a function of dijet invariant mass $m_{12}$ for $b$-tagged (right) dijet production in Au+Au collisions at $\sqrt{s_{N N}}=200 \mathrm{GeV}$ at sPHENIX. We fix $g_{\text {med }}=2.0$, and the band corresponds to a range of masses of the propagating system between $m_{b}$ and $2 m_{b}$. Figures are reproduced from Ref. [10].

and while the shapes in $\mathrm{A}+\mathrm{A}$ and $\mathrm{p}+\mathrm{p}$ reactions are easy to differentiate, the changes in the average momentum imbalance $\left\langle z_{J}\right\rangle$ are subtle, on the order if $10 \%$.

As an example of observable where jet quenching effects "add" rather than "subtract" we look at the dijet invariant mass $m_{12}^{2}=\left(p_{1}+p_{2}\right)^{2}$. It can be written in terms of the jets' transverse momentum and rapidity as follows

$$
m_{12}^{2}=m_{1}^{2}+m_{2}^{2}+2\left[m_{1 T} m_{2 T} \cosh (\Delta \eta)-p_{1 T} p_{2 T} \cos (\Delta \phi)\right]
$$

where $m_{i}^{2}=p_{i}^{2}$ and $m_{i T}=\sqrt{m_{i}^{2}+p_{i T}^{2}}$ are the invariant mass squared and the transverse mass for one of the jets; $\Delta \eta$ and $\Delta \phi$ are the differences in the rapidities and the azimuthal angles, respectively. We can evaluate the dijet mass distribution from the double differential dijet cross section as follows

$$
\frac{d \sigma}{d m_{12}}=\int d p_{1 T} d p_{2 T} \frac{d \sigma}{d p_{1 T} d p_{2 T}} \delta\left(m_{12}-\sqrt{\left\langle m_{1}^{2}\right\rangle+\left\langle m_{2}^{2}\right\rangle+2 p_{1 T} p_{2 T}\langle\cosh (\Delta \eta)-\cos (\Delta \phi)\rangle}\right),
$$

We find that the suppression of the dijet invariant mass can be as large as a factor of 10 at both RHIC and the LHC. For reference, the typical suppression of inclusive jets is typically a factor of 2. The nuclear modification is characterized by enhanced sensitivity to the coupling between the jet and the medium, for example a $10 \%$ variation in $g$ can lead to more than a factor of 2 difference in the suppression, denoted $R_{A A}$. Heavy quark mass effects are also more pronounced. One example is shown in the right panel of Figure 1, where the reduction in $R_{A A}$ toward smaller dijet masses is purely driven by the large heavy quark mass $m_{b}$. The steeply falling spectra at RHIC energies facilitate such differentiation, but dijet masses can also be very effectively studied at the LHC.

\section{Inclusive $b$-jet production from semi-inclusive jet functions}

Inclusive jet production in both proton-proton $(\mathrm{p}+\mathrm{p})$ and heavy ion $(\mathrm{p}+\mathrm{A}, \mathrm{A}+\mathrm{A})$ collisions is 

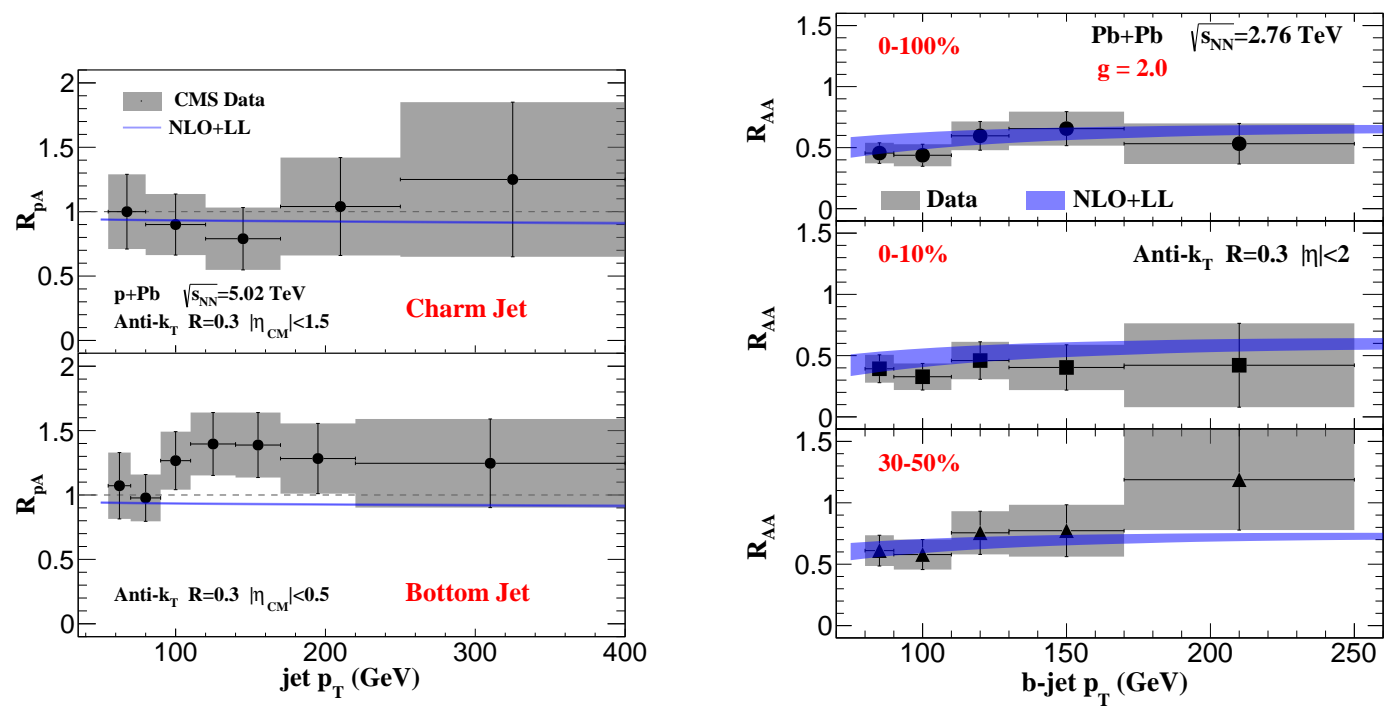

Figure 2: Left: comparison of predicted heavy flavor jet cross section $R_{\mathrm{pA}}$ in proton-nucleus collisions to CMS measurements $[17,18]$ of $c$-jets (top) and $b$-jets (bottom) at $\sqrt{s_{\mathrm{NN}}}=5.02 \mathrm{TeV}$. Right: the nuclear modification factor $R_{A A}$ of $b$-jets for different centrality classes (0-100\%, 0-10\% and 30-50\%), as indicated in the legend. Data is from CMS measurements [6]. Figures are reproduced from Ref. [12].

a multiscale problem, suited to effective field theory treatment. The differential jet cross section versus $p_{T}$ and $\eta$ in hadronic collisions can be expressed as convolution of the parton distribution functions (PDFs), the hard part, and the semi-inclusive jet functions (SiJFs):

$$
\begin{aligned}
\frac{d \sigma_{p p \rightarrow J+X}}{d p_{T} d \eta}= & \frac{2 p_{T}}{s} \sum_{a, b, c} \int_{x_{a}^{\min }}^{1} \frac{d x_{a}}{x_{a}} f_{a}\left(x_{a}, \mu\right) \int_{x_{b}^{\min }}^{1} \frac{d x_{b}}{x_{b}} f_{b}\left(x_{b}, \mu\right) \\
& \times \int_{z_{\min }}^{1} \frac{d z_{c}}{z_{c}^{2}} \frac{d \hat{\sigma}_{a b \rightarrow c}\left(\hat{s}, p_{T} / z_{c}, \hat{\eta}, \mu\right)}{d v d z} J_{J / c}\left(z_{c}, w_{J} \tan \left(R^{\prime} / 2\right), m_{Q}, \mu\right) .
\end{aligned}
$$

Here $f_{a, b}$ are the PDFs, $d \hat{\sigma}_{a b \rightarrow c}\left(\hat{s}, p_{T}, \hat{\eta}, \mu\right) / d v d z$ is the hard function for the sub-process $a b \rightarrow c$, and $J_{J / c}$ is the jet function. It describes the probability of a parton $c$ with transverse momentum $p_{T} / z_{c}$ to fragment into a jet $J$ with $p_{T}$. The $\ln R$ resummation for jet production can be achieved by evolving the SiJFs from the jet scale $\mu_{J}$ to the factorization scale $\mu$. The renormalization group equations are the usual time-like DGLAP evolution equations.

High energy jet production in $\mathrm{p}+\mathrm{A}$ reactions places constraints on cold nuclear matter $(\mathrm{CNM})$ effects. Within the statistical and systematic uncertainties many experimental measurements are consistent with a range of possibilities - from no nuclear effects to $\pm 10 \%$ cross section modification. The model described here takes into account the initial-state CNM energy loss. It is compared to data from 5.02 $\mathrm{TeV} \mathrm{p}+\mathrm{Pb}$ collisions $[17,18]$ in the left panel of Figure 2. In nucleus-nucleus collisions the semi-inclusive jet functions receive corrections from in-medium energy dissipation 
and QGP-induced branching. For example, the NLO medium correction to the $Q \rightarrow J_{Q} \mathrm{SiJF}$ is

$$
\begin{aligned}
J_{J_{Q} / Q}^{\mathrm{med},(1)}\left(z, p_{T} R, m, \mu\right) & =\int_{z(1-z) p_{T} R}^{\mu} d q_{\perp} P_{Q Q}^{\mathrm{med}}\left(z, m, q_{\perp}\right)-\delta(1-z) \int_{0}^{1} d x \int_{x(1-x) p_{T} R}^{\mu} d q_{\perp} P_{Q Q}^{\mathrm{med}}\left(x, m, q_{\perp}\right) \\
& =\left[\int_{z(1-z) p_{T} R}^{\mu} d q_{\perp} P_{Q Q}^{\mathrm{med}}\left(z, m, q_{\perp}\right)\right]_{+} .
\end{aligned}
$$

In the above equation, the terms correspond to the contribution with a radiation outside of the jet cone and the combination of the real radiation inside the jet cone and the virtual loop corrections. $P_{Q Q}^{\text {med }}\left(z, m, q_{\perp}\right)$ is the medium-induced heavy quark splitting kernel.

Numerical calculations of $b$-jet suppression are compared to data [6] in the right panel of Figure 2. $R_{\mathrm{AA}}$ decreases (indicating larger suppression) with increasing collision centrality. The attenuation factor is less dependent on the centrality when compared to the light jet modification. The predictions agree very well with the data for both the inclusive cross sections and the nuclear modification factors.

\section{In-medium splitting functions to any order in opacity}

Advances in the theoretical understanding and experimental measurements of reconstructed jets and heavy flavor necessitate more precise control over in-medium branching processes. An important step in this direction is to obtain in-medium splitting kernels beyond the soft gluon approximation and to higher orders in opacity - the correlation between multiple scattering centers in the nuclear medium. In Ref. [19] a lightcone wavefunction techniques were combined with recurrence relations to allow for the calculation medium-induced splitting processes to an arbitrary order in opacity. One should note that it was argued that full numerical evaluation of such processes is necessary, as corrections to simple analytic limits are large [20].

The original work, which focused on the single $q \rightarrow q g$ chanel, has now ben extended to include all 4 lowest order branching processes in the medium $(q \rightarrow q g, g \rightarrow g g, q \rightarrow g q, g \rightarrow q \bar{q})$. The type of single-Born and double-Born interactions between the propagating partonic system and the nuclear medium that we consider are shown in the left panel of Figure 3. Having derived the universal color and kinematic structure of these interactions, the recursion relations between the components of the splitting functions can be cast in the form of a matrix equation, which has a particularly simple triangular form because of their causal structure. We have written a Mathematica code to solve this equation to any desired order in opacity.

Even though the number of terms in the splitting kernels grows rapidly at higher orders in opacity, we can evaluate these expressions numerically. One should note that the convergence of the VEGAS algorithm that approximates the probability distribution function of the integrand becomes slower and the calculation of the second order corrections uses 10 times larger number of random samples than that used in the leading order calculation. The one-dimensional parton splitting distribution $\frac{d N}{d x}$ is shown in the right panel of Figure 3 for a $100 \mathrm{GeV}$ jet. For light partons the corrections from second order in opacity in the small $x$ and large $x$ regions are all negative and of order $30 \%-70 \%$. In the region of $x \sim 0.5$ the second order correction is positive and boosts the branching probability. This can also be clearly seen in the insets of the figure. For heavy quarks $2^{\text {nd }}$ order in opacity corrections are noticeably smaller in most parts of the available phase space. 

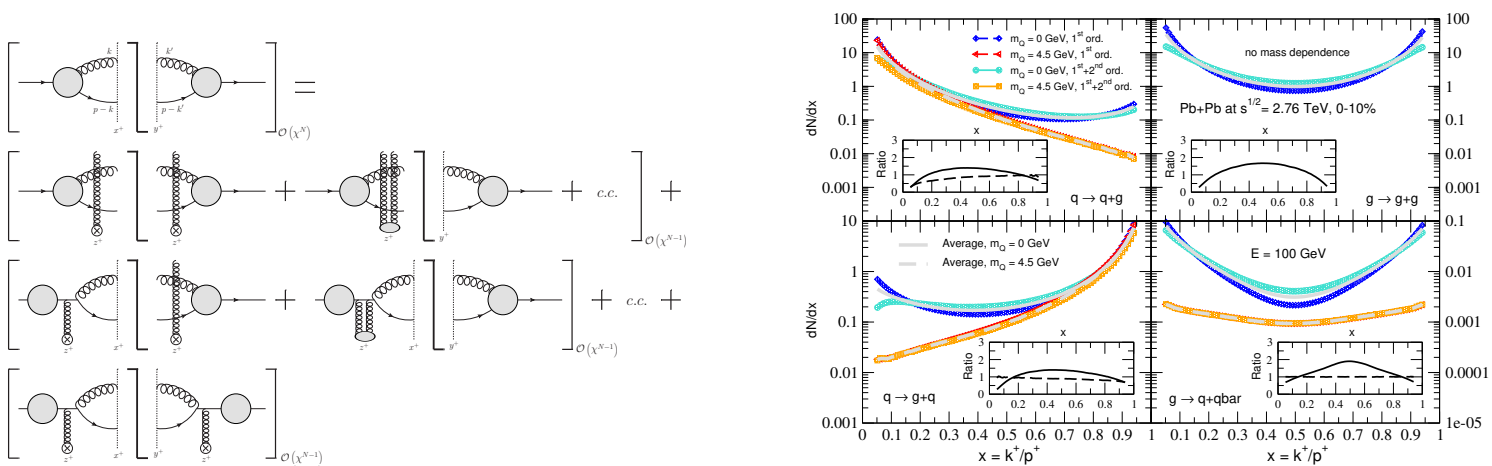

Figure 3: Left: diagrammatic representation of the recursion relation for the Final/Final sector of the $q \rightarrow q g$ branching channel. Right: the one-dimensional differential splitting functions $\frac{d N}{d x}$ for a $100 \mathrm{GeV}$ jet as a function of the splitting fraction $x$. Both light and heavy quark results are shown where relevant. Insets: The ratio of the $\left(1^{\text {st }}+2^{\text {nd }}\right) / 1^{\text {st }}$ order results. Figures are reproduced from Ref. [16]

\section{Conclusions}

In summary, I covered a range of subjects related to the theory and phenomenology of heavy flavor jets in heavy ion reactions - from the formal aspects of parton shower formation to the practical issue of identifying new observables with better sensitivity to jet quenching effects that can enhance the existing tool chest of the field. Heavy flavor jets studies represent the convergence of the now well-developed heavy meson and light jet theory and measurements. I expect that their role as precision diagnostics of nuclear matter will continue to grow in the future, especially as they are being backed up by new theoretical developments.

\section{References}

[1] A. Andronic et al., Heavy-flavour and quarkonium production in the LHC era: from proton-proton to heavy-ion collisions, Eur. Phys. J. C76 (2016) 107 [1506. 03981].

[2] J. Huang, Z.-B. Kang and I. Vitev, Inclusive b-jet production in heavy ion collisions at the LHC, Phys. Lett. B726 (2013) 251 [1306.0909].

[3] F. Senzel, J. Uphoff, Z. Xu and C. Greiner, The different energy loss mechanisms of inclusive and b-tagged reconstructed jets within ultra-relativistic heavy-ion collisions, Phys. Lett. B773 (2017) 620 [1602.05086].

[4] H. T. Li and I. Vitev, Inverting the mass hierarchy of jet quenching effects with prompt b-jet substructure, Phys. Lett. B793 (2019) 259 [1801.00008].

[5] W. Dai, S. Wang, S.-L. Zhang, B.-W. Zhang and E. Wang, Transverse Momentum Balance and Angular Distribution of $b \bar{b}$ Dijets in Pb+Pb collisions, 1806.06332.

[6] CMS collaboration, Evidence of b-Jet Quenching in PbPb Collisions at $\sqrt{s_{N N}}=2.76 \mathrm{TeV}$, Phys. Rev. Lett. 113 (2014) 132301 [1312.4198].

[7] CMS collaboration, Comparing transverse momentum balance of $b$ jet pairs in $p p$ and $\mathrm{PbPb}$ collisions at $\sqrt{s_{\mathrm{NN}}}=5.02 \mathrm{TeV}, \mathrm{JHEP} 03$ (2018) 181 [1802 . 00707]. 
[8] H. Hassan, Charm jet production and properties in $p p, p-\mathrm{Pb}$, and $\mathrm{PbPb}$ collisions measured with ALICE at the LHC, in 19th International Workshop on Charm Physics (CHARM 2018), (Novosibirsk, Russia), May, 2018, https://hal.archives-ouvertes.fr/hal-01846896.

[9] PHENIX collaboration, An Upgrade Proposal from the PHENIX Collaboration, 1501.06197.

[10] Z.-B. Kang, J. Reiten, I. Vitev and B. Yoon, Light and heavy flavor dijet production and dijet mass modification in heavy ion collisions, Phys. Rev. D99 (2019) 034006 [1810. 10007 ].

[11] M. Djordjevic and M. Gyulassy, Heavy quark radiative energy loss in QCD matter, Nucl. Phys. A733 (2004) 265 [nucl-th/0310076].

[12] H. T. Li and I. Vitev, Inclusive heavy flavor jet production with semi-inclusive jet functions: from proton to heavy-ion collisions, 1811.07905.

[13] L. Dai, C. Kim and A. K. Leibovich, Heavy Quark Jet Fragmentation, JHEP 09 (2018) 109 [1805.06014].

[14] Z.-B. Kang, F. Ringer and I. Vitev, Effective field theory approach to open heavy flavor production in heavy-ion collisions, JHEP 03 (2017) 146 [1610 . 02043 ].

[15] C. Lee, P. Shrivastava and V. Vaidya, Predictions for energy correlators probing substructure of groomed heavy quark jets, 1901.09095.

[16] M. D. Sievert, I. Vitev and B. Yoon, A complete set of in-medium splitting functions to any order in opacity, 1903.06170.

[17] CMS collaboration, Measurements of the charm jet cross section and nuclear modification factor in pPb collisions at $\sqrt{s_{N N}}=5.02 \mathrm{TeV}$, Phys. Lett. B772 (2017) 306 [1612.08972].

[18] CMS collaboration, Transverse momentum spectra of inclusive b jets in pPb collisions at $\sqrt{s_{N N}}=$ 5.02 TeV, Phys. Lett. B754 (2016) 59 [1510.03373].

[19] M. D. Sievert and I. Vitev, Quark branching in QCD matter to any order in opacity beyond the soft gluon emission limit, Phys. Rev. D98 (2018) 094010 [1807.03799].

[20] X. Feal and R. Vazquez, Intensity of gluon bremsstrahlung in a finite plasma, Phys. Rev. D98 (2018) 074029 [1811.01591]. 